\title{
LEAST SQUARES APPROXIMATION OF LYAPUNOV EXPONENTS*
}

\author{
BY
}

B. S. BERGER AND M. ROKNI

University of Maryland, College Park, Maryland

\begin{abstract}
Discrete least squares approximations are shown to converge for the Lyapunov exponents of dynamical systems. Numerical examples demonstrate the approximation's utility.

Introduction. The limit explicit in the definition of Lyapunov exponents, L.E., (3), (4), has been found through the evaluation of $\ln \lambda_{\mathrm{n}}$ and $q(t)$ and formation of the ratio $\ln \lambda_{\mathrm{n}} / q(t)$ for increasing $t$, [2], [4], and [6]. It is shown, in the following, that this limit may be expressed as $\lim _{t \rightarrow \infty} a_{2}$, where $a_{2}$ is a coefficient in the least squares approximation of $\ln \lambda_{n}$. The definition of L.E. used here includes the customary definition as a special case with $q(t) \equiv t$. The L.E., $\beta$, for dynamical systems in which $\ln \lambda_{\mathbf{n}} \sim \beta \ln t, t \rightarrow \infty$, [8], (12), is found through a suitable choice of approximating functions. $\sim \equiv$ asymptotic equivalence. A numerical study indicates the utility of the derived algorithms.
\end{abstract}

Lyapunov exponents. Consider a continuous dynamical system

$$
\dot{\mathbf{x}}=\mathbf{f}(\mathbf{x}, t)
$$

where $\mathbf{x}$ are spatial curvilinear coordinates in an $n$-dimensional Euclidean phase space, $E_{n}$. Under general conditions the solution of (1), [1],

$$
\mathbf{x}=\mathbf{x}(\mathbf{X}, t)
$$

is one-to-one, continuous together with its inverse and continuously differentiable with respect to $\mathbf{X}$ and $t$. $\mathbf{X}$ are material coordinates.

A subspace, $V_{m}$, of dimension $m<n$ may be defined by the parametric equation $\mathbf{X}=\mathbf{X}(\mathbf{u})$ in material coordinates and, after the deformation (2), by $\mathbf{x}=\mathbf{x}(\mathbf{u})$ in spatial coordinates. $\mathbf{u}$ has $m$ components. Let $d a_{(m)}$ and $d A_{(m)}$ be spatial and material area elements of $V_{m}$ respectively. The $m$-dimensional L.E. associated with a trajectory originating at $\mathbf{X}$ is defined as, [2], [3],

$$
\chi\left(\mathbf{X}, V_{m}\right) \equiv \lim _{t \rightarrow \infty} \sup \left(\ln \left(d a_{m} / d A_{m}\right)\right) / q(t)
$$

where $q(t) \equiv t$. The one-dimensional L.E. for the arc, $d s$, with tangent, $\mathbf{n}$, associated with a trajectory having initial conditions respectively of $d S, \mathbf{N}, \mathbf{X}$ is defined as, [2],

$$
\chi(\mathbf{X}, \mathbf{n}) \equiv \lim _{t \rightarrow \infty} \sup \left(\ln \lambda_{\mathbf{n}}\right) / q(t)
$$

*Received October 19, 1988. 
where $\lambda_{\mathrm{n}} \equiv d s / d S,(d s)^{2}=g_{i j} d x^{i} d x^{j},(d S)^{2}=G_{I J} d X^{I} d X^{J}$ and $q(t) \equiv t$. In the following, only exact L.E. are considered and therefore sup will be omitted, [2].

Since $D\left(d A_{m}\right) / D t=0$ and $D\left(d A^{I_{1} \cdots I_{m}}\right) / D t=0$, (3) may be expressed as, [4],

$$
\chi\left(\mathbf{X}, V_{m}\right)=\lim _{t \rightarrow \infty}(1 / q) \int_{t_{0}}^{t}(1 / m !) \frac{1}{d a_{(m)}} \frac{D}{D t}\left(d a^{i_{1} \cdots i_{m}}\right) \frac{1}{d a_{(m)}} d a_{i_{1} \cdots i_{m}} d t
$$

where $d a^{i_{1} \cdots i_{m}}$ is the spatial area tensor and

$$
\frac{1}{d a_{(m)}} \frac{D}{D t} d a^{i_{1} \cdots i_{m}}=v^{i_{1}}, r \frac{1}{d a_{(m)}} d a^{r i_{2} \cdots i_{m}}+\cdots+v^{i_{m}}, r \frac{1}{d a_{(m)}} d a^{i_{1} i_{2} \cdots i_{m-1} r} .
$$

Denote $d a_{m} / d A_{m}$ or $\lambda_{\mathrm{n}}$ by $h(t)$. If $q(t)=t$ and $\ln h(t) \sim \alpha t$ then the limit in (3) or (4) is $\alpha$. If $\ln h(t) \sim \beta \ln t, t \rightarrow \infty$, then the limits in (3) or (4) vanish, [5]. In this case, if $q(t)=\ln t$, then the limit in (3) or (4) is $\beta$. Subsequently, the coefficient, $a_{2}$, in a least squares approximation of $f(t) \equiv \ln h(t)$ is shown to converge to the limit $\alpha$ or $\beta$.

Least squares approximation. Given an approximating function, $G(t)=a_{1}+a_{2} q(t)$, where $a_{1}$ and $a_{2}$ are constants. For a least squares approximation of $f(t), a_{2}$ is determined by

$$
a_{2}=\left(N \sum f_{i} q_{i}-\left(\sum f_{i}\right)\left(\sum q_{i}\right)\right) / A,
$$

where $f_{i} \equiv f\left(t_{i}\right), A \equiv N \sum q_{i}^{2}-\left(\sum q_{i}\right)^{2}$ and $\sum \equiv$ summation on $i=1, N$ discrete values of $t$, [7]. It follows that if $t_{i}=\Delta \cdot i, q(t) \equiv t$, and $\lim _{t \rightarrow \infty}(f(t) / t)=\alpha$ then $\lim _{N \rightarrow \infty} a_{2}=\alpha$. This may be shown by setting $t_{i}=\Delta \cdot i$ in (7) and noting that $\sum i=\left(N^{2}+N\right) / 2$ and $\sum i^{2}=N^{3} / 3+N^{2} / 2+N / 6$. (7) becomes

$$
a_{2}=(12 / \Delta)\left(\sum\left(i f_{i}\right) /\left(N\left(N^{2}-1\right)\right)-\sum f_{i} /(2 N(N-1))\right) .
$$

By assumption $\lim _{t \rightarrow \infty}(f(t) / t)=\alpha$. Then $\lim _{i \rightarrow \infty} Z_{i}=0$ where $Z_{i}=\left(f_{i} / t_{i}\right)-\alpha$. It follows that given any $\varepsilon>0$ then $\exists N_{\varepsilon} \ni\left|Z_{i}\right|<\varepsilon$ for all $i>N_{\varepsilon}$. Substituting $f_{i}=\left(Z_{i}+\alpha\right) i \cdot \Delta$ into the second and third quotients in (8) and taking the limit as $N \rightarrow \infty$ gives $\Delta \alpha / 3$ and $\Delta \alpha / 4$ respectively. Substituting these expressions into (8) yields the result.

Consider the case in which $q(t)=\ln t, h(t)=C t^{\beta}(1+g(t)), C>0$ is a constant, $g(t)$ is bounded, $g(t)>-1$ and $\lim _{t \rightarrow \infty} g(t)=0$. Setting $t_{i}=\Delta \cdot i$ and $f(t)=$ $\beta \ln t+\ln C(1+g(t))$ in (7) gives

$$
a_{2}=\beta+\frac{1}{A}\left[N \sum\left(\ln i \ln C\left(1+g_{i}\right)\right)-\sum \ln i \sum \ln C\left(1+g_{i}\right)\right]
$$

where $A=N \sum(\ln i)^{2}-(\ln N !)^{2}$. Note that $\ln N !=\left(N+\frac{1}{2}\right) \ln (N+1)-N-1+f_{2}(N+1)$, $\left|f_{2}(N+1)\right|<1, \ln (1+g) \leq g$ for $0 \leq g<1,|\ln (1-g)| \leq 3 g / 2$ for $0 \leq g \leq 0.58$, $\ln (N+1)=\ln N+2\left(\frac{1}{2 N+1}+\frac{1}{3}\left(\frac{1}{2 N+1}\right)^{3}+\cdots\right)$, [7], $\sum \ln i=\ln N$ ! and $\sum \ln ^{2} i=$ $N\left(\ln ^{2} N-2 \ln N+2\right)+\ln ^{2} N(1+0(1))$ as $N \rightarrow \infty$. The last relationship follows from the Euler-Maclaurin summation formula, [12] and [13]. It follows that $A \sim N^{2}$ and the second term on the right hand side of (9) vanishes as $N \rightarrow \infty$. Then $\lim _{N \rightarrow \infty} a_{2}=\beta$. 
Computation of L.E. The Lorenz and Rossler equations together with an equation given by Davis, [8], are subsequently used as test cases:

Lorenz equations, [9],

$$
\begin{array}{ll}
\dot{x}_{1}=a\left(x_{2}-x_{1}\right) & a=16.0, \\
\dot{x}_{2}=x_{1}\left(b-x_{3}\right)-x_{2} & b=45.92, \\
\dot{x}_{3}=x_{1} x_{2}-c x_{3} & c=4.0 ;
\end{array}
$$

Rossler equations, [10],

$$
\begin{array}{ll}
\dot{x}_{1}=-\left(x_{2}+x_{3}\right) & a=0.15, \\
\dot{x}_{2}=x_{1}+a x_{2} & b=0.20, \\
\dot{x}_{3}=b+x_{3}\left(x_{1}-c\right) & c=10.0 ;
\end{array}
$$

Davis equations, [8],

$$
\begin{aligned}
& \dot{x}_{1}=x_{2} \\
& \dot{x}_{2}=\left(1.5 x_{2}^{2}-k x_{1}^{4}\right) / x_{1} \quad k=2 .
\end{aligned}
$$

For the given set of parameters $(10),(11)$ possess chaotic solutions. The solution to (12) is given in [8] as $x_{1}=a b\left((t+p)^{2}+b^{2}\right)^{-1}$ with $a^{2}=2 / k$. If $d v$ is a volume element of the phase space then it may be shown that, [11], $\ln d v=\int v_{k, k} d t$ where $v_{k} \equiv \dot{x}_{k}$. For (12)

$$
\ln d v=-3\left(\ln \left(\left((t+p)^{2}+b^{2}\right) /\left(p^{2}+b^{2}\right)\right)\right)
$$

and $\lim _{t \rightarrow \infty}(\ln d v / \ln t)=-6$. It follows that the sum of the L.E. of $(12), \chi_{1}+\chi_{2}=$ -6 , and -8.656 if $\log _{2}$ is used in (13) rather than $\ln ,[6]$.

A fourth order Runge-Kutta integrator was used to solve (10), (11), and (12) with $\Delta t=0.01$. The L.E. were found, $[2,4,6,14]$, through the evolution of $\ln \lambda_{n}, q(t)$ and the ratio $\ln \lambda_{n} / q(t)$, subsequently referred to as Case 1 , and the evolution of $a_{2}$, with $\Delta=0.1,(8)$ or (9), referred to as Case 2. For (10) with $t=100$ and $q(t)=t$, Case 1 gave L.E. $=2.03, .0139,-32.348$ while Case 2 gave $2.18,-.0045,-32.48$. The corresponding results for $t=1000$ were $2.15, .002,-32.455$, and $2.16,-.0008$, -32.457 respectively. Reference [6] reports values of $2.15, .003$, and -32.45 . The initial convergence of Case 2 appears to have been more rapid than that of Case 1 .

For the Rossler equation, (11), with $q(t)=t$, L.E. for Cases 1 and 2 were 0.126 , $-.0003,-14.2$, and $0.127,-.00025,-14.1$ respectively. Reference [6] reports values of $0.13,0.00$, and -14.1 .

L.E. for Cases 1 and 2, (12), with $q(t)=\ln t, p=.1, b=.1$, and $t=200$ were $\chi_{1}=-3.633, \chi_{2}=-8.191, \chi_{1}+\chi_{2}=-11.825$, and $\chi_{1}=-2.916, \chi_{2}=-5.692$, $\chi_{1}+\chi_{2}=-8.607$ respectively. The exact value of $\chi_{1}+\chi_{2}=-8.656,(13)$, which is in good agreement with $\chi_{1}+\chi_{2}=-8.607$ from Case $2 . \chi_{1}+\chi_{2}=-11.825$ for Case 1 which differs considerably from the exact value.

Conclusions. Numerical tests of the least squares algorithms for the computation of L.E. demonstrate their convergence rates to be equal to and in some instances, (12), greater than those of previous methods. The poor convergence of Case 1 for (12) is attributable to the slow convergence to zero of the ratio of the term $3 \cdot \ln \left(p^{2}+b^{2}\right)$, (13), and $\ln t$. The rate of convergence of the least squares algorithm, Case 2, is not siginificantly affected by this term. 
Acknowledgment. The authors acknowledge the encouragement of Dr. Matthew Gordon-Clark and the assistance of C. J. Berger. Computations were supported by the Computer Science Center of the University of Maryland.

\section{REFERENCES}

[1] J. Hale, Ordinary Differential Equations, Krieger Pub. Co., 1982

[2] G. Benettin, L. Galagani, A. Giorgilli, and J. Strelcyn, Lyapunov characteristic exponents for smooth dynamical systems and for Hamiltonian systems; method for computing all of them, Meccanica 15, 9-30 (1980)

[3] V. I. Oseledec, A multiplicative ergodic theorem, Lyapunov characteristic numbers for dynamical systems, Trans. Moscow Math. Soc. 19, 197-231 (1968)

[4] M. Rokni and B. S. Berger, Lyapunov exponents and subspace evolution, Quart. Appl. Math. 45, 789-793 (1987)

[5] L. Cesari, Asymptotic Behavior and Stability Problems in Ordinary Differential Equations, Springer, 1971

[6] A. Wolf, B. Swift, H. L. Swinney, and J. A. Vastand, Determining Lyapunov exponents from time series, Physica 16D, 285-317 (1985)

[7] M. Abramowitz and I. Stegun, Handbook of Mathematical Functions, NBS, 55, 1965

[8] H. T. Davis, Introduction to Nonlinear Differential and Integral Equations, Dover Pub., 1962

[9] E. N. Lorenz, Deterministic nonperiodic flow, J. Atm. Sci. 20, 130-141 (1963)

[10] O. E. Rossler, An equation for continuous chaos, Phys. Lett. 57A, 397-398 (1976)

[11] B. S. Berger and M. Rokni, Lyapunov exponents and continuum kinematics, Int. J. Engrg. Sci. 25, 1251-1257 (1987)

[12] N. G. De Bruijn, Asymptotic Methods in Analysis, North Holland Pub. Co., 1961

[13] F. W. J. Olver, Asymptotics and Special Functions, Academic Press, 1974

[14] B. S. Berger and M. Rokni, Lyapunov exponents and the evolution of normals, Int. J. Engrg. Sci. 25, 1393-1396 (1987) 